\title{
Association between TXNRD1 polymorphisms and anti-tuberculosis drug-induced hepatotoxicity in a prospective study
}

\author{
G.Y. Ji ${ }^{1}$, Y. Wang ${ }^{1}$, S.Q. Wu ${ }^{1}$, Q.Q. Liu ${ }^{2}$, J.C. Wu ${ }^{1}$, M.M. Zhang', \\ A.J. Sandford ${ }^{3}$ and J.Q. He ${ }^{1}$ \\ ${ }^{1}$ Department of Respiratory and Critical Care Medicine, West China Hospital, \\ Sichuan University, Chengdu, Sichuan, China \\ ${ }^{2}$ Department of Laboratory Medicine, West China Hospital, Sichuan University, \\ Guoxue Alley, Chengdu, Sichuan, China \\ ${ }^{3}$ Centre for Heart Lung Innovation, \\ University of British Columbia and St. Paul's Hospital, Vancouver, BC, Canada \\ Corresponding author: J.Q. He \\ E-mail: 18980602293@qq.com
}

Genet. Mol. Res. 15 (3): gmr.15038296

Received December 16, 2015

Accepted January 2, 2016

Published September 2, 2016

DOI http://dx.doi.org/10.4238/gmr.15038296

Copyright (C) 2016 The Authors. This is an open-access article distributed under the terms of the Creative Commons Attribution ShareAlike (CC BY-SA) 4.0 License.

\begin{abstract}
Anti-tuberculosis drug-induced hepatotoxicity (ATDH) is a serious adverse reaction to anti-tuberculosis (TB) treatment. Thioredoxin reductase 1 (TXNRD1), encoded by the TXNRD1 gene, is an important enzyme involved in oxidant challenge. TXNRD1 plays a key role in regulating cell growth and transformation, and protects cells against oxidative damage. We investigated the association between TXNRD1 polymorphisms and ATDH susceptibility. In this prospective study, 280 newly diagnosed TB patients were followed-up for 3 months after beginning anti-TB therapy. Tag single-nucleotide polymorphisms (tag-SNPs) of TXNRD1 were selected using Haploview 4.2 based on
\end{abstract}


the HapMap database of the Chinese Han in Beijing (CHB) panel. Genotyping was performed using the MassARRAY platform. Of the 280 patients enrolled in this study, 33 were lost to follow-up, 24 had ATDH, and 223 were free from ATDH. After adjusting for sex, age, smoking status, and body mass index, there were no significant differences in the allele and genotype frequency distributions of TXNRD1 SNPs between the ATDH and non-ATDH groups (all $\mathrm{P}>0.05$ ). The haplotype analysis showed that haplotype TCAGCC was associated with an increased risk of ATDH susceptibility [P $=0.024$, OR $(95 \% \mathrm{CI})=6.273$ (1.023-38.485)]. Further stratified analyses showed that the haplotype TCAGCC was associated with ATDH susceptibility in female subjects $[\mathrm{P}=0.036, \mathrm{OR}(95 \% \mathrm{CI})=5.711(0.917-35.560)]$ and non-smokers $[\mathrm{P}$ $=0.029, \mathrm{OR}(95 \% \mathrm{CI})=6.008(0.971-37.158)]$. Our results suggest that TXNRD1 variants may favor ATDH susceptibility in females and nonsmokers. Further studies are required to verify this association.

Key words: Thioredoxin reductase 1; TXNRD1; Polymorphism; Anti-tuberculosis drug-induced hepatotoxicity; ATDH

\section{INTRODUCTION}

Tuberculosis (TB) is a threat to people's health and has been recognized as a worldwide health problem, with 13.0 million prevalent TB cases, 9.6 million new TB cases, and 1.3 million deaths in 2014 (World Health Organization, 2015). For controlling the TB epidemic, a standard anti-TB treatment protocol including isoniazid, rifampin, pyrazinamide, ethambutol, and/or streptomycin for 6-9 months has been recommended (World Health Organization, 2002). However, these anti-TB drugs usually have some adverse drug reactions (ADRs) (Chen et al., 2015). One of the most common and serious ADRs is anti-tuberculosis drug-induced hepatotoxicity (ATDH), which can lead to non-adherence, drug resistance, or treatment failure (Kaona et al., 2004). Therefore, a better understanding of the risk factors of ATDH development would be helpful. Previous studies have shown that the risk factors can be classified as non-genetic and genetic factors (Chen et al., 2015). Non-genetic factors include female sex, race, and environmental factors such as alcohol intake (Pande et al., 1996; Lee et al., 2002; Marra et al., 2007; de Castro et al., 2010). The genetic factors are involved in drug metabolism, transport, and immune and antioxidant responses, and include $\mathrm{N}$-acetyltransferase 2 (NAT2), BTB and CNC homology $1(B A C H 1)$, tumor necrosis factor- $\alpha(T N F)$ and adenosine triphosphate binding cassette B1 (ABCB1) (Yimer et al., 2011; Kim et al., 2012; Nanashima et al., 2012; Du et al., 2013).

Thioredoxin reductase 1 (TXNRD1), encoded by the TXNRD1 gene, is an important NADPH-dependent enzyme with broad substrate specificity related to oxidant challenge (Kwon et al., 2012). TXNRD1 plays a key role in regulating cell growth and transformation, and protecting cells against oxidative damage (Kwon et al., 2012). Previous studies have found that there is a strong association between oxidative stress and liver disease, and the TXNRD1-mediated antioxidant function protects the liver from oxidative injury (Minami et al., 2005; Zidek et al., 2007; Cederbaum et al., 2009). Kwon et al. (2012) reported that the genetic variations in TXNRD1 favor the development of drug-induced liver injury (DILI).

Genetics and Molecular Research 15 (3): gmr.15038296 
However, there has been no study on the association between TXNRD1 polymorphisms and ATDH susceptibility. Therefore, in this study we aimed to investigate the associations between TXNRD1 polymorphisms and ATDH among the Chinese Han population.

\section{MATERIAL AND METHODS}

\section{Study subjects}

In this study, 280 newly diagnosed TB patients were recruited before receiving antiTB therapy between August 2012 and March 2015 in the West China Hospital of Sichuan University. All TB patients were diagnosed by specialist physicians based on respiratory symptoms, typical infiltrate findings on chest radiographs, bacteriological evidence (sputum smear or culture), or histopathological evidence (ATS, 2000). Patients with hepatitis B virus (HBV), hepatitis C virus (HCV), or human immunodeficiency virus (HIV), those who drank habitually, had liver disease or abnormal liver function before using anti-TB drugs, those who were concurrently using hepatotoxic drugs, and those lost to follow-up, were excluded.

All subjects took rifampicin $600 \mathrm{mg}$ ( or $450 \mathrm{mg}$ if body weight was $<50 \mathrm{~kg}$ ), isoniazid $300 \mathrm{mg}$, ethambutol $750 \mathrm{mg}$, and pyrazinamide $1500 \mathrm{mg}$ every day for 2 months. After 2 months of treatment, pyrazinamide was discontinued and the other three drugs were continued for a total of 6 months.

We performed liver function tests routinely at 2 weeks, 1 month, 2 months, and 3 months from the start of treatment, or whenever the subjects displayed any notable manifestations of liver dysfunction, such as loss of appetite or jaundice. We adopted diagnostic criteria for ATDH based on the International Consensus Meeting, as follows: 1) an increase of over two fold above the upper limit of normal (ULN) in the level of alanine aminotransferase (ALT) with/without $\geq$ two times the ULN in the level of aspartate aminotransferase (AST) and total bilirubin during the anti-TB therapy period (Bénichou, 1990); and 2) the result of causality assessment was certain, probable, or possible (World Health Organization, 2005; Corsini and Bortolini, 2013).

\section{Ethics statement}

Each subject was required to provide written informed consent. The Ethics Committee of the West China Hospital of Sichuan University, China, provided authorization for our study.

\section{Selection/genotyping of TXNRD1 single-nucleotide polymorphisms (SNPs)}

TXNRD1 is located on chromosome 12. SNPs were selected from 3000 base pairs downstream to 3000 base pairs upstream of the TXNRD1 gene. According to the HapMap Chinese Han in Beijing (CHB) database (http://hapmap.ncbi.nlm.nih.gov), with minor allele frequency (MAF) $\geq 0.05$ and $\mathrm{r}^{2} \geq 0.80$, we chose six tag single-nucleotide polymorphisms (tag-SNPs) of TXNRD1 (rs10735393, rs4964287, rs4595619, rs4246270, rs4246271, and rs11611385) for further genotyping.

Peripheral venous blood samples were collected from all patients in ethylenediaminetetraacetic acid (EDTA) tubes (BD Vacutainers, Franklin Lakes, NJ, USA). Genomic DNA was extracted using a Genomic DNA Extraction kit (Axygen Scientific

Genetics and Molecular Research 15 (3): gmr.15038296 
Inc., Union City, CA, USA). Selected SNPs of TXNRD1 were genotyped by the Sequenom MassARRAY iPLEX platform using polymerase chain reaction (PCR) and matrix-assisted laser desorption/ionization time-of-flight (MALDI-TOF) mass spectrometry.

\section{Statistical analysis}

SPSS software and the SHEsis system (http://analysis.Bio-x.cn/SHEsisMain.htm) were adopted for statistical analysis. The SNPs and distributions of demographic characteristics between the ATDH and non-ATDH groups were calculated by the chi-squared test (categorical data) or the $t$-test (numerical data). Linkage disequilibrium (LD) between SNPs and haplotype analyses were examined using the SHEsis program. Agreement with the Hardy-Weinberg equilibrium (HWE) was assessed using the chi-squared test. Odds ratios (ORs) and 95\% confidence intervals $(95 \% \mathrm{CIs})$ were calculated by logistic regression analysis adjusting for confounding factors including sex, age, smoking status, and body mass index (BMI). P values $<0.05$ were considered statistically significant.

\section{RESULTS}

\section{Demographic and clinical characteristics of study subjects}

Of the 280 subjects initially recruited to this study, we excluded 33 subjects: 19 were lost to follow-up; 12 had used other hepatotoxic medications, had complications from diseases influencing liver function, or were unable to provide samples for liver function tests in follow-up; and 2 individuals failed to respond to first-line anti-TB treatment and switched to second-line anti-TB therapy before completing 3 months of therapy. The remaining 247 TB patients completed 3 months of first-line anti-TB treatment and follow-up. Of those 247 subjects, $24(9.8 \%)$ patients with levels of ALT/AST/total bilirubin more than twice the ULN were enrolled into the ATDH group, and the remaining 223 (90.2\%) individuals were put in the non-ATDH group. The demographic characteristics of the study population are listed in Table 1. No significant differences were found in gender, age, smoking status, or BMI between the ATDH and non-ATDH groups.

\section{Table 1. Characteristics of patients in the anti-tuberculosis drug-induced hepatotoxicity (ATDH) and non-} ATDH groups.

\begin{tabular}{l|c|c|c}
\hline Characteristic & Patients with ATDH $(\mathrm{N}=24)$ & Patients without ATDH $(\mathrm{N}=223)$ & P value \\
\hline Gender $(\mathrm{male} /$ female $)$ & $8 / 16$ & $90 / 133$ & 0.502 \\
\hline Age $(\text { years })^{\mathrm{a}}$ & $32.79 \pm 11.96$ & $38.91 \pm 16.00$ & 0.070 \\
\hline Weight $(\mathrm{kg})^{\mathrm{a}}$ & $54.86 \pm 8.69$ & $54.67 \pm 10.30$ & 0.932 \\
\hline BMI $\left(\mathrm{kg} / \mathrm{m}^{2}\right)^{\mathrm{a}}$ & $21.58 \pm 2.93$ & $20.53 \pm 3.74$ & 0.217 \\
\hline Smoking history & $6(25.0 \%)$ & $63(28.3 \%)$ & 0.717 \\
\hline Baseline value & & & \\
\hline ALT $(\mathrm{U} / \mathrm{L})^{\mathrm{b}}$ & $23(15-36)$ & $17(11-24)$ & 0.095 \\
\hline AST $(\mathrm{U} / \mathrm{L})^{\mathrm{b}}$ & $22(20-34)$ & $22(18-27)$ & 0.147 \\
\hline Total bilirubin $(\mu \mathrm{M})^{\mathrm{b}}$ & $11.4(8.4-13.5)$ & $10.2(7.6-14.1)$ & 0.950 \\
\hline During treatment $($ peak value) & & & $24(17-34)$ \\
\hline ALT $(\mathrm{U} / \mathrm{L})^{\mathrm{b}}$ & $172(111-255)$ & $26(20-34)$ & $<0.0001$ \\
\hline AST $(\mathrm{U} / \mathrm{L})^{\mathrm{b}}$ & $83(69-201)$ & $9.1(6.2-12.6)$ & $<0.0001$ \\
\hline Total bilirubin $(\mu \mathrm{M})^{\mathrm{b}}$ & $12.8(7.7-19.3)$ & 0.073
\end{tabular}

BMI = body mass index; ALT $=$ alanine aminotransferase; AST $=$ aspartate aminotransferase. ${ }^{\text {a}}$ Values are reported as mean $\pm \mathrm{SD}$ (range). ${ }^{b}$ Values are reported as median (inter-quartile range). 


\section{Association between TXNRD1 SNPs and ATDH}

Six tag-SNPs (rs10735393, rs4964287, rs4595619, rs4246270, rs4246271, and rs11611385) of TXNRD1 were selected and successfully genotyped in our study. The genotypic frequencies of these SNPs in the ATDH and the non-ATDH groups are shown in Table 2. As shown in Table 2, we analyzed the data with three different genetic models: allelic, codominant, and dominant. After adjusting for confounding factors including gender, age, smoking status, and BMI, there were no statistically significant differences (all $\mathrm{P}>0.05$ ) between the two groups. Using SHEsis software, the linkage disequilibrium (LD) across the SNPs was calculated with both $\mathrm{r}^{2}$ and D' (Figure 1). We performed further haplotype analysis by excluding the rare haplotypes (lowest frequency threshold $=0.03$ ) $($ Table 3 ). The analysis results showed that the TCAGCC haplotype increased the risk of ATDH susceptibility [P= 0.024 , OR $(95 \% \mathrm{CI})=6.273(1.023-38.485)]$.

Table 2. Association between TXNRD1 single-nucleotide polymorphisms (SNPs) and anti-tuberculosis druginduced hepatotoxicity (ATDH).

\begin{tabular}{|c|c|c|c|c|c|c|c|c|c|c|}
\hline \multirow[t]{2}{*}{ SNPs } & \multirow[t]{2}{*}{ Groups } & \multicolumn{3}{|c|}{ Subjects with genotype, $\mathrm{N}(\%)$} & \multicolumn{2}{|c|}{ Allelic model } & \multicolumn{2}{|c|}{ Codominant model } & \multicolumn{2}{|c|}{ Dominant model } \\
\hline & & 00 & 01 & 11 & OR $(95 \% \mathrm{CI})^{\mathrm{a}}$ & $\mathrm{P}^{\mathrm{a}}$ value & $\mathrm{OR}(95 \% \mathrm{CI})^{\mathrm{a}}$ & $\mathrm{P}^{\mathrm{a}}$ value & OR $(95 \% \mathrm{Cl})^{\mathrm{a}}$ & $\mathrm{P}^{\mathrm{a}}$ value \\
\hline rs 10735393 & n-ATDH & $153(69.9 \%)$ & $56(25.6 \%)$ & $10(4.6 \%)$ & & & & & & \\
\hline $\mathrm{T}>\mathrm{G}$ & DH & $15(62.5 \%)$ & $8(33.3 \%)$ & $1(4.2 \%)$ & $1.408(0.651-3.045)$ & 0.385 & $1.872(0.704-4.982)$ & 0.209 & $1.708(0.667-4.374)$ & 0.264 \\
\hline rs4964287 & n-ATDI & $129(57.8 \%)$ & $75(33.6 \%)$ & $19(8.5 \%)$ & & & & & & \\
\hline $\mathrm{C}>\mathrm{T}$ & $\overline{\mathrm{DH}}$ & $15(62.5 \%)$ & $8(33.3 \%)$ & $1\left(4.2^{\circ}\right.$ & $0.765(0.334-1.752)$ & 0.527 & $0.805(0.285-2.272)$ & 0.681 & $0.753(0.281-2.017)$ & 0.573 \\
\hline $\mathrm{rs} 4595$ & n-AT & & $95(4$. & 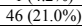 & & & & & & \\
\hline $\mathrm{G}>\mathrm{A}$ & $\mathrm{DH}$ & $7\left(29.2^{\circ}\right)$ & $12(50.0$ & $\frac{5(20.8}{5(20)}$ & $1.174(0.611-2.257)$ & 0.630 & $1.236(0$ & 0.697 & $3.389)$ & 0.622 \\
\hline rs 4246270 & ATDH & & $55(2)$ & & & & & & & \\
\hline $\mathrm{A}>\mathrm{G}$ & DH & 15( & $8(33$ & $1(4$. & $1.425(0.637-3.186)$ & 0.388 & $1.602(0.589-4.356)$ & 0.355 & $1.579(0.604-4.130)$ & 0.352 \\
\hline rs 42462 & non-ATI & 157( & $55(2$ & & & & & & & \\
\hline $\mathrm{G}>\mathrm{C}$ & & 15( & & & $1.412(0.631-3.156)$ & 0.401 & $1.586(0.583-4.313)$ & 0.366 & $1.562(0.597-4.088)$ & 0.363 \\
\hline rs 116 & non-ATDH & 146( & $60(27.1 \%)$ & $15(6.8$ & & & & & & \\
\hline $\mathrm{C}>\mathrm{T}$ & ATDH & $15(62.5 \%)$ & $8(33.3 \%)$ & $1(4.2 \%)$ & $1.011(0.437-2.341)$ & 0.980 & $1.152(0.403-3.296)$ & 0.792 & $1.074(0.397-2.908)$ & 0.888 \\
\hline
\end{tabular}

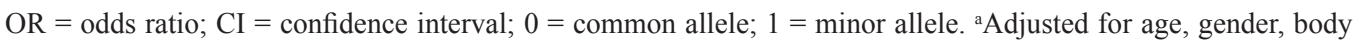
mass index (BMI), and smoking in a logistic regression model. For OR calculations, each group was compared with the non-ATDH group.

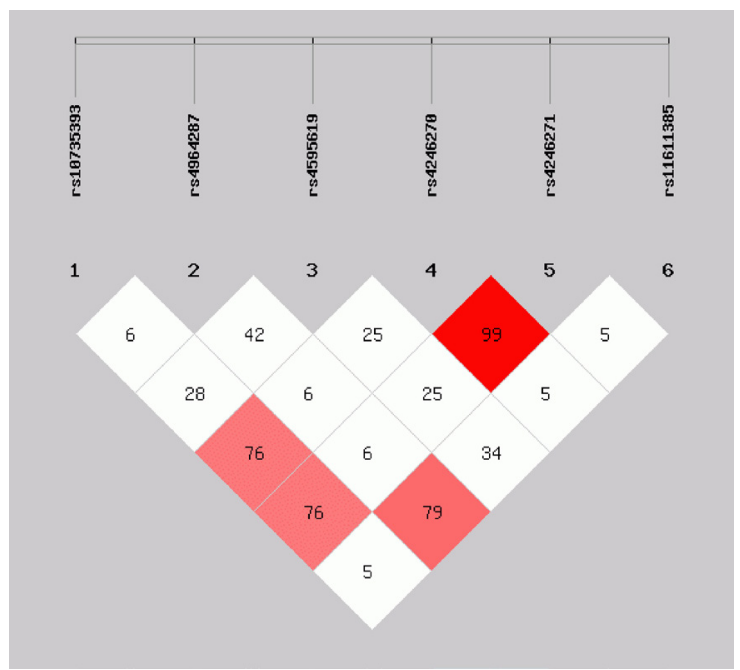

Figure 1. Linkage disequilibrium (LD) of TXNRD1 single nucleotide polymorphisms. The $\mathrm{r}^{2}$ values are displayed on the squares.

Genetics and Molecular Research 15 (3): gmr.15038296 
Table 3. Frequencies of haplotypes consisting of TXNRD1 single-nucleotide polymorphisms (SNPs).

\begin{tabular}{l|l|c|c|c|c}
\hline Haplotypes & Stratification variables & Cases, N $(\%)$ & Controls, N $(\%)$ & OR $(95 \% \mathrm{CI})$ & P value \\
\hline GCAAGC & All & $2.0(4.2 \%)$ & $9.0(2.1 \%)$ & $2.068(0.434-9.860)$ & 0.352 \\
\hline & Female & $2.0(6.3 \%)$ & $4.0(1.5 \%)$ & $4.267(0.750-24.287)$ & 0.076 \\
\hline & Non-smoker & $2.0(5.6 \%)$ & $6.0(1.9 \%)$ & $2.990(0.581-15.401)$ & 0.17 \\
\hline GCAGCC & All & $8.0(16.7 \%)$ & $65.99(15.1 \%)$ & $1.124(0.504-2.510)$ & 0.775 \\
\hline & Female & $6.0(18.7 \%)$ & $41.0(15.8 \%)$ & $1.233(0.478-3.182)$ & 0.665 \\
\hline TCAGCC & Non-smoker & $6.0(16.7 \%)$ & $49.0(15.7 \%)$ & $1.070(0.423-2.706)$ & 0.887 \\
\hline & All & $2.0(4.2 \%)$ & $3.0(0.7 \%)$ & $6.273(1.023-38.485)$ & 0.024 \\
\hline & Female & $2.0(6.3 \%)$ & $3.0(1.2 \%)$ & $5.711(0.917-35.560)$ & 0.036 \\
\hline TCGAGC & Non-smoker & $2.0(5.6 \%)$ & $3.0(1.0 \%)$ & $6.008(0.971-37.158)$ & 0.029 \\
\hline & All & $26.0(54.2 \%)$ & $251.0(57.3 \%)$ & $0.876(0.481-1.593)$ & 0.664 \\
\hline TTAAGT & Female & $15.0(46.9 \%)$ & $149.0(57.3 \%)$ & $0.657(0.315-1.373)$ & 0.262 \\
\hline & Non-smoker & $18.0(50.0 \%)$ & $179.0(57.4 \%)$ & $0.737(0.369-1.471)$ & 0.386 \\
\hline & All & $10.0(20.8 \%)$ & $90.0(20.5 \%)$ & $1.015(0.487-2.114)$ & 0.969 \\
\hline & Female & $7.0(21.9 \%)$ & $52.0(20.0 \%)$ & $1.120(0.459-2.732)$ & 0.803 \\
\hline
\end{tabular}

$\mathrm{TB}=$ tuberculosis; $\mathrm{OR}=$ odds ratio; $\mathrm{CI}=$ confidence interval.

\section{Comparison of the association between TXNRD1 SNPs and ATDH stratified by gender}

Previous studies have shown that gender is important in the pharmacokinetics of drugs (Tanaka and Hisawa, 1999; Morris et al., 2003). Therefore, we further investigated the association between TXNRD1 SNPs and ATDH susceptibility stratified by gender (Table 4). After adjusting for confounding factors including gender, age, smoking status, and BMI, there were no significant differences between the two groups in the male and female subgroups. We also performed haplotype analysis in the two subgroups. There was a statistically significant difference in females but not in males. As shown in Table 3, the OR for the TCAGCC haplotype as a risk factor in females was $5.711(95 \% \mathrm{CI}=0.917-35.560, \mathrm{P}=0.036)$.

\section{Comparison of the association between TXNRD1 SNPs and ATDH stratified by smoking status}

In the subgroup analysis stratified by gender, we found that the TCAGCC haplotype demonstrated an increased risk of ATDH susceptibility in female subjects. Furthermore, among the 149 female patients in our study, 145 (97.3\%) individuals were non-smokers. Therefore, to fully explore whether there was a relationship between TXNRD1 SNPs and ATDH in non-smokers, we further analyzed the TXNRD1 data stratified by smoking status (Table 4). No statistically significant differences were found between the ATDH and nonATDH groups in the smoking and non-smoking subgroups. Further haplotype analysis showed that the TCAGCC haplotype increased the risk of ATDH in non-smokers but not in smokers [P $=0.029, \mathrm{OR}(95 \% \mathrm{CI})=6.008(0.971-37.158)]$.

\section{DISCUSSION}

ATDH is one of the most common and serious ADRs (Shang et al., 2011). Individuals show obvious differences in ADRs. Therefore, a better understanding of the predisposing factors of ATDH susceptibility is crucial for guiding individualized treatment. Studies have shown that the predisposing factors of susceptibility to ATDH mainly comprise environmental and genetic factors, especially the latter (Chen et al., 2015). These genetic factors, such as

Genetics and Molecular Research 15 (3): gmr.15038296 
Table 4. Association between TXNRD1 single-nucleotide polymorphisms (SNPs) and anti-tuberculosis druginduced hepatotoxicity (ATDH) stratified by gender and smoking status.

\begin{tabular}{|c|c|c|c|c|c|c|c|c|c|c|c|}
\hline \multirow[t]{2}{*}{ SNPs } & \multirow{2}{*}{\begin{tabular}{|l|}
$\begin{array}{l}\text { Stratification } \\
\text { variables }\end{array}$ \\
\end{tabular}} & \multirow[t]{2}{*}{ Groups } & \multicolumn{3}{|c|}{ Subjects with genotype, $\mathrm{N}(\%)$} & \multicolumn{2}{|l|}{ Allelic model } & \multicolumn{2}{|c|}{ Codominant model } & \multicolumn{2}{|c|}{ Dominant model } \\
\hline & & & 00 & 01 & 11 & OR $(95 \% \mathrm{CI})^{\mathrm{a}}$ & $\mathrm{P}^{\mathrm{a}}$ value & OR $(95 \% \mathrm{Cl})^{\mathrm{a}}$ & $\begin{array}{l}\mathrm{Pa}^{\mathrm{a}} \text { value } \\
\end{array}$ & OR $(95 \% \mathrm{CI})^{\mathrm{a}}$ & $\mathrm{P}^{\mathrm{p}}$ value \\
\hline rs10735393 & \begin{tabular}{|l} 
Female \\
\end{tabular} & \begin{tabular}{|l|} 
non-ATDH \\
\end{tabular} & $92(70.8 \%)$ & $31(23.8 \%)$ & $7(5.4 \%)$ & & & & & & \\
\hline \multirow[t]{7}{*}{$\mathrm{T}>\mathrm{G}$} & & ATDH & $9(56.2 \%)$ & $6(37.5)$ & $1(6.2 \%)$ & $1.604(0.653-3.938)$ & 0.303 & $2.260(0.684-7.472)$ & 0.181 & $2.064(0.659-6.465)$ & 0.214 \\
\hline & \begin{tabular}{|l|l|} 
Male \\
\end{tabular} & 1-ATDH & $61(68.5 \%)$ & $25(28.1 \%)$ & $3(3.4 \%)$ & & & & & & \\
\hline & & ATDH & $6(75 \%)$ & $2(25.0 \%)$ & $0(0)$ & $882(0.175-4.457)$ & 0.880 & $1.215(0.197-7.491)$ & 0.834 & $1.043(0.171-6.347)$ & 0.964 \\
\hline & \begin{tabular}{|l|} 
Smoker \\
\end{tabular} & non-ATDH & $44(69.8 \%)$ & $18(28.6 \%)$ & $1(1.6 \%)$ & & & & & & \\
\hline & & ATDH & $4(66.7 \%)$ & $2(33.3 \%)$ & $0(0)$ & $1.280(0.209-7.828)$ & 0.790 & $2.487(0.253-24.485)$ & 0.435 & $.944(0.210-17.988)$ & 0.558 \\
\hline & \begin{tabular}{|l|} 
Non-smoker \\
\end{tabular} & non-ATDI & $109(69.9 \%)$ & $38(24.4 \%)$ & $9(5.8 \%)$ & & & & & & \\
\hline & & AT & $11(61.1 \%)$ & $6(33.3$ & $1(5.6$ & $1.390(0.582-3.320)$ & 459 & $1.837(0.592-5.705)$ & & $1.677(0.572-4.918)$ & 0.346 \\
\hline \multirow{8}{*}{$\mathrm{C}>\mathrm{T}$} & \begin{tabular}{|l|} 
Female \\
\end{tabular} & \begin{tabular}{|l|} 
non-ATDH \\
\end{tabular} & $78(58.6 \%)$ & $45(33$. & $10(7.5)$ & & & & & & \\
\hline & & ATDH & $10(62.5 \%)$ & $5(31.2 \%)$ & $1(6.2 \%)$ & $091(0.405-2.938)$ & 863 & $0.930(0.256-3.371)$ & 0.911 & \begin{tabular}{|l|}
$0.995(0.297-3.332)$ \\
\end{tabular} & 0.994 \\
\hline & Male & \begin{tabular}{|l|} 
non-ATDH \\
\end{tabular} & $51(56.7 \%)$ & $30(33.3 \%)$ & $9(10.0 \%)$ & & & & & & \\
\hline & & \begin{tabular}{|l|} 
ATDH \\
\end{tabular} & $5(62.5 \%)$ & $3(37.5 \%)$ & $0(0)$ & $0.561(0.114-2.751)$ & 0.476 & $0.824(0.135-5.024)$ & 0.833 & $0.653(0.107-3.972)$ & 0.643 \\
\hline & \begin{tabular}{|l|} 
Smoker \\
\end{tabular} & non & $35(54.7 \%)$ & $22(34$. & $7(10.9 \%$ & & & & & & \\
\hline & & $\mathrm{A} 7$ & $4(66$. & $2(33$ & $0(0)$ & $484(0.051-4.546)$ & 525 & $662(0.055-7.973)$ & 745 & $.533(0.044$ & 622 \\
\hline & \begin{tabular}{|l|} 
Non-smoker \\
\end{tabular} & \begin{tabular}{|l|} 
non-ATDH \\
\end{tabular} & $94(59.1 \%)$ & $53(33$ & $12(7.5 \%)$ & & & & & & \\
\hline & & ATDH & $11(61.1 \%)$ & $6(33.3 \%)$ & $1(5.6 \%)$ & $874(1.432-2.668)$ & 877 & $1.040(1.326-3.319)$ & .947 & \begin{tabular}{|l|}
$1.056(0.350-3.184)$ \\
\end{tabular} & 0.923 \\
\hline rs 4595619 & Female & \begin{tabular}{|l|} 
non-ATDH \\
\end{tabular} & $48(36.9 \%)$ & $53(40.8 \%)$ & $29(22.3 \%)$ & & & & & & \\
\hline \multirow[t]{7}{*}{$\mathrm{G}>\mathrm{A}$} & & \begin{tabular}{|l|} 
ATDH \\
\end{tabular} & $4(25.0 \%)$ & $7(43.8 \%)$ & $5(31.2 \%)$ & $1.699(0.761-3.796)$ & 0.196 & $1.155(0.271-4.925)$ & 0.845 & \begin{tabular}{|l|}
$1.638(0.465-5.774)$ \\
\end{tabular} & 0.443 \\
\hline & Male & & $30(33.7 \%)$ & $42(4$ & $17(19$. & & & & & & \\
\hline & & & $3(3$ & & $0(0$ & 8) & 0.518 & 7) & 0.504 & 61) & 0903 \\
\hline & \begin{tabular}{|l} 
Smoker \\
\end{tabular} & \begin{tabular}{|l|} 
non-ATDH \\
\end{tabular} & $21(33.3 \%)$ & $30(47.6 \%)$ & $12(19.0 \%)$ & & & & & & \\
\hline & & ATDH & $2(33.3 \%)$ & $4(66.7 \%)$ & $0(0)$ & $33(0.166-4.174)$ & 824 & $2.142(0.173-26.469)$ & 0.553 & \begin{tabular}{|l}
$1.696(0.134-21.514)$ \\
\end{tabular} & 0.684 \\
\hline & \begin{tabular}{|l|} 
Non-smoker \\
\end{tabular} & \begin{tabular}{|l|} 
non-ATDH \\
\end{tabular} & $57(36.5 \%)$ & $65(41.7 \%)$ & $34(21.8 \%)$ & & & & & & \\
\hline & & \begin{tabular}{|l|} 
ATDH \\
\end{tabular} & $5(27.8 \%)$ & $8(44.4$ & $5(27.8 \%)$ & $1.479(0.701-3.119)$ & 0.304 & $1.229(0.337-4.487)$ & 0.755 & $1.494(0.476-4.688)$ & 0.491 \\
\hline $\mathrm{rs} 4246$ & $\begin{array}{l}\text { Female } \\
\end{array}$ & & & & $7(5$. & & & & & & \\
\hline \multirow{7}{*}{$\mathrm{A}>\mathrm{G}$} & & & $9(56.2)$ & $6(37$. & $1(6.2 \%$ & 6) & 0.477 & 0) & 10 & 75) & 0.419 \\
\hline & Male & \begin{tabular}{|l|} 
non-ATDH \\
\end{tabular} & $65(73.0 \%)$ & $23(25.8$ & $1\left(1.1^{\circ}\right.$ & & & & & & \\
\hline & & & $6(75.0$ & $2(25$. & $0(0$ & $1.387(0.259-7.418)$ & 0.702 & $1.504(0.226-10.010)$ & 0.673 & \begin{tabular}{|l}
$1.504(0.226-10.010)$ \\
\end{tabular} & 0.673 \\
\hline & \begin{tabular}{|l|} 
Smoker \\
\end{tabular} & $\mathrm{H}$ & & & & & & & & & \\
\hline & & \begin{tabular}{|l}
$\mathrm{A}^{2}$ \\
\end{tabular} & $4(66.7)$ & $2(33$. & 00( & $1.956(0.290-13.196)$ & 0.491 & $2.651(0.237-29.706)$ & 0.429 & $2.651(0.237-29.706)$ & 0.429 \\
\hline & No & & & & & & & & & & \\
\hline & & & & & $1(5$. & & & & & & \\
\hline rs 4246271 & \begin{tabular}{|l} 
Female \\
\end{tabular} & 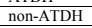 & & 32( & $7(5$. & & & & & & \\
\hline \multirow[t]{7}{*}{$\mathrm{G}>\mathrm{C}$} & & & & & & $1.383(0.545-3.509)$ & 0.4 & $1.642(0.486-5.544)$ & 0.424 & \begin{tabular}{|l|}
$1.582(0.501-4.997)$ \\
\end{tabular} & 0.435 \\
\hline & \begin{tabular}{|l|l} 
Male \\
\end{tabular} & & & & $1(1$. & & & & & & \\
\hline & & & 6 & $2(2)$ & 0( & $1.387(0.259-7.418)$ & 0.702 & $1.504(0.226-10.010)$ & 0.673 & $1.504(0.226-10.010)$ & 0.673 \\
\hline & \begin{tabular}{|l|} 
Smoker \\
\end{tabular} & . & & $17(2)$ & 0( & & & & & & \\
\hline & & & & & 0 & . & & & & 06) & 0.429 \\
\hline & \begin{tabular}{|l|} 
Non-smoker \\
\end{tabular} & & & & & & & & & & \\
\hline & & & & & & $1.274(0.511-3.178)$ & & $1.421(0.440-4.589)$ & 0.557 & \begin{tabular}{|l|}
$1.394(0.460-4.224)$ \\
\end{tabular} & 0.557 \\
\hline \multirow{8}{*}{$\begin{array}{l}\mathrm{rs} 11611385 \\
\mathrm{C}>\mathrm{T}\end{array}$} & Female & no & & & $8(6$. & & & & & & \\
\hline & & ATDH & $10(62.5 \%)$ & $5\left(31.2^{\circ}\right.$ & $1(6.2 \%)$ & $1.548(0.560-4.281)$ & 0.399 & $1.421(0.382-5.280)$ & 0.600 & \begin{tabular}{|l}
$1.528(0.445-5.248)$ \\
\end{tabular} & 0.500 \\
\hline & $\mathrm{M}_{\mathrm{i}}$ & non- & & $24(2$ & $7(7.9$ & & & & & & \\
\hline & & & & & 0 & $0.706(0.143-3$. & & $1.081(0.178-6$ & 933 & 54) & 0.87 \\
\hline & \begin{tabular}{|l} 
Smoker \\
\end{tabular} & & & & & & & & & & \\
\hline & & & 4 & & 0 & $0.646(0.068-6.135)$ & 0.704 & $0.889(0.075-10.521)$ & 0.925 & \begin{tabular}{|l|}
$0.746(0.063-8.878)$ \\
\end{tabular} & 0.816 \\
\hline & \begin{tabular}{|l|} 
Non-smoker \\
\end{tabular} & \begin{tabular}{|l|} 
non-ATI \\
\end{tabular} & $106(67.1 \%)$ & $42(26.6 \%)$ & $10(6.3 \%)$ & & & & & & \\
\hline & & ATDH & $11(61.1 \%)$ & $6(33.3 \%)$ & $1(5.6 \%)$ & $1.450(0.572-3.675)$ & 0.433 & $1.512(0.465-4.918)$ & 0.492 & \begin{tabular}{|l|}
$1.541(0.500-4.744)$ \\
\end{tabular} & 0.451 \\
\hline
\end{tabular}

$\mathrm{OR}=$ odds ratio; $\mathrm{CI}=$ confidence interval; $0=$ common allele; $1=$ minor frequency allele. ${ }^{\mathrm{a}}$ Adjusted for age, gender, and smoking in a logistic regression model. For OR calculations, each group was compared with the control group.

$N A T 2, B A C H 1, T N F$, and $A B C B 1$, are involved in the pharmacokinetics of drugs including metabolism, transport, and immune and antioxidant responses (Yimer et al., 2011; Kim et al., 2012; Nanashima et al., 2012; Du et al., 2013). Studies have shown that TXNRD1mediated antioxidant function protects the liver against oxidative injury, and the genetic variations in TXNRD1 are related to DILI (Minami et al., 2005; Zidek et al., 2007; Kwon et al., 2012). However, there have not been any reports on the association between TXNRD1 polymorphisms and ATDH susceptibility. Therefore, the aim of our study was to explore the genetic associations between TXNRD1 polymorphisms and ATDH susceptibility.

Six tag-SNPs (rs10735393, rs4964287, rs4595619, rs4246270, rs4246271, and rs11611385) were selected for this research. Our findings showed that there were no statistically significant differences in the allele and genotype frequency distributions of TXNRD1 SNPs between the ATDH and non-ATDH groups (all P > 0.05). In further haplotype analysis, the TCAGCC haplotype showed an increased risk of ATDH susceptibility. Previous studies have shown that gender plays an important role in the pharmacokinetics of drugs (Tanaka and Hisawa, 1999; Morris et al., 2003). Therefore, we further investigated the association between TXNRD1 SNPs and ATDH susceptibility stratified by gender. We found that only the

Genetics and Molecular Research 15 (3): gmr.15038296 
TCAGCC haplotype demonstrated a statistically significant difference in the risk of susceptible to ATDH among female individuals. No statistically significant differences were found in the male subgroup analysis. The vast majority of females included in our study were nonsmokers. Therefore, we speculated that TXNRD1 may be associated with ATDH susceptibility in non-smokers. Thus, we further analyzed the TXNRD1 data stratified by smoking status. There was a statistically significant difference in the non-smokers, but not in the smokers. The TCAGCC haplotype exhibited a risk of ATDH susceptibility in the non-smoking subgroup. Our results suggest that TXNRD1 variants may favor ATDH susceptibility in female nonsmokers. Previous studies have shown that ATDH is more common among females (Lee et al., 2002; Marra et al., 2007). Kwon et al. (2012) found that there were associations between the genetic variations of TXNRD1 and DILI. Other studies have shown that TXNRD1-mediated antioxidant function protects the liver against oxidative injury (Minami et al., 2005; Zidek et al., 2007). Thus, our results are in accordance with previous findings from the literature.

The strengths of our study are as follows. First, this is the first study on the association between TXNRD1 SNPs and ATDH susceptibility. The TCAGCC haplotype showed an increased risk of susceptible to ATDH. This result provides evidence of the association between TXNRD1 SNPs and ATDH susceptibility in the Chinese Han population. Furthermore, there was a different correlation in the female subgroup. Therefore, it can be speculated that there is an association between TXNRD1 and non-smoking. Almost all the female subjects were non-smokers. The statistically significant difference is more obvious in the non-smokers than in the female subgroup analysis. Therefore, there may be a female non-smoking effect, with non-smoking having a greater influence. Second, our study was a prospective study and was less affected by certain biases such as recall bias.

The limitations of our study are as follows. First, the study population was limited to Chinese Han subjects. Relevant research that includes other nationalities is needed to fully explore the association between TXNRD1 SNPs and ATDH. Second, the sample size was small, particularly the ATDH group, partially owing to the strict inclusion criteria. Thus, larger sample studies should be performed to verify the findings.

In conclusion, our results indicated that the TCAGCC haplotype is associated with increased risk of ATDH susceptibility. Our study is the first prospective study of TXNRD1 SNPs and ATDH susceptibility. The results suggest that TXNRD1 variants may favor the pathogenesis of ATDH in Chinese Han individuals. Large-scale studies on different ethnic populations are required to verify the findings.

\section{Conflicts of interest}

The authors declare no conflict of interest.

\section{ACKNOWLEDGMENTS}

Research supported by the National Natural Science Foundation of China (Grants \#81170042 and \#81370121).

\section{REFERENCES}

American Thoracic Society (ATS) (2000). Diagnostic standards and classification of tuberculosis in adults and children. Am. J. Respir. Crit. Care Med. 161: 1376-1395. http://dx.doi.org/10.1164/ajrccm.161.4.16141

Genetics and Molecular Research 15 (3): gmr.15038296 
Bénichou C (1990). Criteria of drug-induced liver disorders. Report of an international consensus meeting. J. Hepatol. 11: 272-276. http://dx.doi.org/10.1016/0168-8278(90)90124-A

Cederbaum AI, Lu Y and Wu D (2009). Role of oxidative stress in alcohol-induced liver injury. Arch. Toxicol. 83: 519548. http://dx.doi.org/10.1007/s00204-009-0432-0

Chen R, Wang J, Zhang Y, Tang S, et al. (2015). Key factors of susceptibility to anti-tuberculosis drug-induced hepatotoxicity. Arch. Toxicol. 89: 883-897. http://dx.doi.org/10.1007/s00204-015-1473-1

Corsini A and Bortolini M (2013). Drug-induced liver injury: the role of drug metabolism and transport. J. Clin. Pharmacol. 53: 463-474. http://dx.doi.org/10.1002/jcph.23

de Castro L, do Brasil PE, Monteiro TP and Rolla VC (2010). Can hepatitis B virus infection predict tuberculosis treatment liver toxicity? Development of a preliminary prediction rule. Int. J. Tuberc. Lung Dis. 14: 332-340.

Du H, Chen X, Fang Y, Yan O, et al. (2013). Slow N-acetyltransferase 2 genotype contributes to anti-tuberculosis druginduced hepatotoxicity: a meta-analysis. Mol. Biol. Rep. 40: 3591-3596. http://dx.doi.org/10.1007/s11033-012$2433-\mathrm{y}$

Kaona FA, Tuba M, Siziya S and Sikaona L (2004). An assessment of factors contributing to treatment adherence and knowledge of TB transmission among patients on TB treatment. BMC Public Health 4: 68. http://dx.doi. org/10.1186/1471-2458-4-68

Kim SH, Kim SH, Yoon HJ, Shin DH, et al. (2012). TNF- $\alpha$ genetic polymorphism -308G/A and antituberculosis druginduced hepatitis. Liver Int. 32: 809-814. http://dx.doi.org/10.1111/j.1478-3231.2011.02697.x

Kwon JW, Shin ES, Lee JE, Kim SH, et al.; Adverse Drug Reaction Research Group in Korea (2012). Genetic variations in TXNRD1 as potential predictors of drug-induced liver injury. Allergy Asthma Immunol. Res. 4: 132-136. http:// dx.doi.org/10.4168/aair.2012.4.3.132

Lee AM, Mennone JZ, Jones RC and Paul WS (2002). Risk factors for hepatotoxicity associated with rifampin and pyrazinamide for the treatment of latent tuberculosis infection: experience from three public health tuberculosis clinics. Int. J. Tuberc. Lung Dis. 6: 995-1000.

Marra F, Marra CA, Bruchet N, Richardson K, et al. (2007). Adverse drug reactions associated with first-line antituberculosis drug regimens. Int. J. Tuberc. Lung Dis. 11: 868-875.

Minami K, Saito T, Narahara M, Tomita H, et al. (2005). Relationship between hepatic gene expression profiles and hepatotoxicity in five typical hepatotoxicant-administered rats. Toxicol. Sci. 87: 296-305. http://dx.doi.org/10.1093/ $\underline{\text { toxsci/kfi235 }}$

Morris ME, Lee HJ and Predko LM (2003). Gender differences in the membrane transport of endogenous and exogenous compounds. Pharmacol. Rev. 55: 229-240. http://dx.doi.org/10.1124/pr.55.2.1

Nanashima K, Mawatari T, Tahara N, Higuchi N, et al. (2012). Genetic variants in antioxidant pathway: risk factors for hepatotoxicity in tuberculosis patients. Tuberculosis (Edinb.) 92: 253-259. http://dx.doi.org/10.1016/j. tube.2011.12.004

Pande JN, Singh SP, Khilnani GC, Khilnani S, et al. (1996). Risk factors for hepatotoxicity from antituberculosis drugs: a case-control study. Thorax 51: 132-136. http://dx.doi.org/10.1136/thx.51.2.132

Shang P, Xia Y, Liu F, Wang X, et al. (2011). Incidence, clinical features and impact on anti-tuberculosis treatment of anti-tuberculosis drug induced liver injury (ATLI) in China. PLoS One 6: e21836. http://dx.doi.org/10.1371/journal. pone. 0021836

Tanaka E and Hisawa S (1999). Clinically significant pharmacokinetic drug interactions with psychoactive drugs: antidepressants and antipsychotics and the cytochrome P450 system. J. Clin. Pharm. Ther. 24: 7-16. http://dx.doi. org/10.1046/j.1365-2710.1999.00200.x

World Health Organization (2015). Global tuberculosis report 2015. http://www.who.int/tb/publications/global_report/ en/ (accessed Nov 2, 2015).

World Health Organization (WHO) (2002). An expanded DOTS framework for effective tuberculosis control. Int. J. Tuberc. Lung Dis. 6: 378-388.

World Health Organization (2005). The use of theWHO-UMC system for standardized case causality assessment. The Uppsala Monitoring Centre, Uppsala.

Yimer G, Ueda N, Habtewold A, Amogne W, et al. (2011). Pharmacogenetic \& pharmacokinetic biomarker for efavirenz based ARV and rifampicin based anti-TB drug induced liver injury in TB-HIV infected patients. PLoS One 6: e27810. http://dx.doi.org/10.1371/journal.pone.0027810

Zidek N, Hellmann J, Kramer PJ and Hewitt PG (2007). Acute hepatotoxicity: a predictive model based on focused illumina microarrays. Toxicol. Sci. 99: 289-302. http://dx.doi.org/10.1093/toxsci/kfm131

Genetics and Molecular Research 15 (3): gmr.15038296 